\title{
Digital Biomarkers
}

\section{Digital}

Health

\section{0}

CiteScore: $\mathbf{6 . 8}$

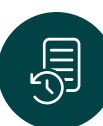

Time to first decision 33 days

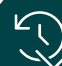

Time to final decision 11 weeks
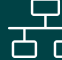

PubMed Central

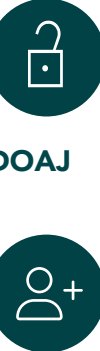

New Editor

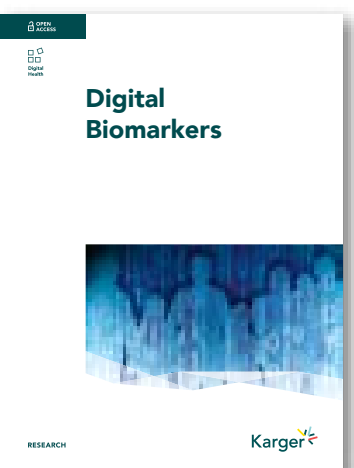

OP OPEN

- ACCESS
Editor-in-Chief

Roozbeh Ghaffari (Evanston, IL)

\section{Bridging computer science and biomedicine}

Digital biomarkers are defined as objective, quantifiable physiological and behavioral data that are collected and measured by means of digital devices such as portables, wearables, implantables, or digestibles. The data collected are typically used to explain, influence, and/or predict health-related outcomes. Digital biomarkers also represent an opportunity to capture clinically meaningful, objective data. Multidisciplinary by design, this innovative Open Access journal bridges the disciplines of computer science, engineering, biomedicine, regulatory science, and informatics. The editorial board includes leaders from academia, life sciences, technology, and government, thus reflecting the broad scope of the journal. Papers are published within 60 days of submission, and the inclusion of videos or other visual materials is supported. Moreover, Digital Biomarkers provides and supports access to data, algorithms, and app repositories linked to the articles published in the journal.

editorialoffice_dib@ karger.com karger.com/dib

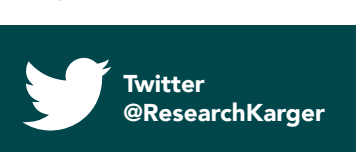

